\title{
Investigación-docencia interdisciplinaria, gestión el conocimiento y tecnología
} Interdisciplinary research-teaching, knowledge management and technology Yira Muñoz-Sánchez ${ }^{a}$, María A. Alonso-Lavernia ${ }^{b}$, Iliana Castillo-Pérez ${ }^{b}$, Verónica Martínez-Lazcano ${ }^{b}$

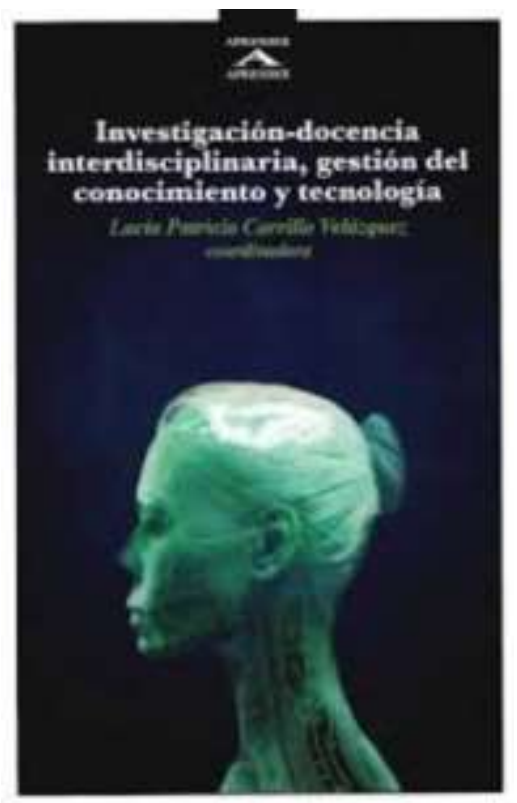

Es un libro que sirve para concientizarse sobre el papel clave que juega de la educación superior en la atención de los diversos problemas de la sociedad en los ámbitos de la misma educación, salud, pobreza, medio ambiente, etc. en donde los avances en las ciencias computacionales y las Tecnologías de la Información y Comunicaciones (TIC) han venido a transformar la sociedad a lo que es hoy en día y la cual presentan numerosos retos por alcanzar.

\footnotetext{
a Autor de Correspondencia, Universidad Autónoma del Estado de Hidalgo, Escuela Superior de Ciudad Sahagún, https://orcid.org/00000002-4876-2747, Email: yira@uaeh.edu.mx

${ }^{b}$ Universidad Autónoma del Estado de Hidalgo, Instituto de Ciencias Básicas e Ingeniería, https://orcid.org/0000-0002-9839-8250, Email: marial@uaeh.edu.mx

c Universidad Autónoma del Estado de Hidalgo, Instituto de Ciencias Básicas e Ingeniería, https://orcid.org/0000-0002-8130-9231, Email: ilianac@uaeh.edu.mx

${ }^{d}$ Universidad Autónoma del Estado de Hidalgo, Instituto de Ciencias Básicas e Ingeniería, https://orcid.org/0000-0003-2172-4000, Email: vlazcano@uaeh.edu.mx
} 
Dichos retos incluyen nuevos escenarios educativos, investigaciones, demandas y procesos, para los cuales se plantea una propuesta teórico-metodológica que permita la operación de procesos que involucran a la investigación y la docencia interdisciplinaria, bajo un modelo constructivista y haciendo uso de la ingeniería de software que hagan posible la comunicación entre varias disciplinas del conocimiento.

Se plantea el trabajo colaborativo entre la investigación y la docencia como una opción viable para solucionar problemas sociales a través de la gestión del conocimiento y soportado por las TIC. Gracias a éstas es posible realizar análisis de redes y protestas sociales.

Finalmente, se comparten experiencias sobre investigación y docencia de tipo interdisciplinaria, gestión del conocimiento y tecnología, para su análisis y futura toma de decisiones.

\section{Referencias}

Carrillo, L. P. (2015). Investigación-docencia interdisciplinaria, gestión del conocimiento y tecnología. México, D.F., México: Universidad Nacional Autónoma de México. 